\title{
Assessment of renal function in mice with unilateral ureteral obstruction using ${ }^{99 \mathrm{~m}} \mathrm{Tc}-\mathrm{MAG} 3$ dynamic scintigraphy
}

Mohammed N Tantawy ${ }^{1,2}$, Rosie Jiang ${ }^{2}$, Feng Wang ${ }^{1,2}$, Keiko Takahashi $^{2}$, Todd E Peterson ${ }^{1}$, Dana Zemel ${ }^{2}$, Chuan-Ming Hao ${ }^{2}$, Hiroki Fujita ${ }^{3}$, Raymond C Harris ${ }^{2,4}$, Christopher C Quarles ${ }^{1,2}$ and Takamune Takahashi ${ }^{2,4^{*}}$

\begin{abstract}
Background: Renal scintigraphy using ${ }^{99 \mathrm{~m}} \mathrm{Tc}$-mercaptoacetyltriglycine $\left({ }^{99 \mathrm{~m}} \mathrm{Tc}-\mathrm{MAG} 3\right)$ is widely used for the assessment of renal function in humans. However, the application of this method to animal models of renal disease is currently limited, especially in rodents. Here, we have applied ${ }^{99 \mathrm{~m}} \mathrm{Tc}-\mathrm{MAG} 3$ renal scintigraphy to a mouse model of unilateral ureteral obstruction $(\cup \cup O)$ and evaluated its utility in studying obstructive renal disease.

Methods: UUO mice were generated by complete ligation of the left ureter. Sham-operated mice were used as a control. Renal function was investigated on days $0,1,3$, and 6 post-surgery using dynamic planar imaging of ${ }^{99 m}$ Tc-MAG3 activity following retro-orbital injection. Time-activity curves (TACS) were produced for individual kidneys and renal function was assessed by 1 ) the slope of initial ${ }^{99 m}$ Tc-MAG3 uptake (SIU), which is related to renal perfusion; 2) peak activity; and 3) the time-to-peak (TTP). The parameters of tubular excretion were not evaluated in this study as ${ }^{99 m}$ Tc-MAG3 is not excreted from UUO kidneys.

Results: Compared to sham-operated mice, SIU was remarkably (>60\%) reduced in UUO kidneys at day 1 post surgery and the TACs plateaued, indicating that ${ }^{99 \mathrm{~m} T c-M A G 3}$ is not excreted in these kidneys. The plateau activity in UUO kidneys was relatively low ( 40\% of sham kidney's peak activity) as early as day 1 post surgery, demonstrating that uptake of ${ }^{99 \mathrm{~m} T c-M A G 3}$ is rapidly reduced in UUO kidneys. The time to plateau in UUO kidneys exceeded $200 \mathrm{sec}$, suggesting that ${ }^{99 \mathrm{~m}}$ Tc-MAG3 is slowly up-taken in these kidneys. These changes advanced as the disease progressed. SIU, peak activity and TTPs were minimally changed in contra-lateral kidneys during the study period.

Conclusions: Our data demonstrate that renal uptake of ${ }^{99 \mathrm{~m}} \mathrm{Tc}-\mathrm{MAG} 3$ is remarkably and rapidly reduced in UUO kidneys, while the changes are minimal in contra-lateral kidneys. The parametric analysis of TACs suggested that renal perfusion as well as tubular uptake is reduced in UUO kidneys. This imaging technique should allow non-invasive assessments of UUO renal injury and enable a more rapid interrogation of novel therapeutic agents and protocols.
\end{abstract}

Keywords: Nuclear imaging, Renal scintigraphy, ${ }^{99 m}$ Tc-MAG3, Mouse model, Unilateral ureteral obstruction, Renal function

\footnotetext{
* Correspondence: takamune.takahashi@vanderbilt.edu

${ }^{2}$ Vanderbilt O'Brien Mouse Kidney Physiology and Disease Center, Vanderbilt

University Medical Center, Nashville, TN, USA

${ }^{4}$ Division of Nephrology and Hypertension, Vanderbilt University School of

Medicine, S-3223, MCN, Nashville, TN 37232, USA

Full list of author information is available at the end of the article
} 


\section{Background}

There is considerable interest and potential value in making available imaging methods that permit noninvasive assessments of renal function in animals. Renal scintigraphy or renograms using ${ }^{99 \mathrm{~m}}$ Tc-mercaptoacetyltriglycine ( $\left.{ }^{99 \mathrm{~m}} \mathrm{Tc}-\mathrm{MAG} 3\right)$ is widely used for the assessment of renal function in humans, especially for the evaluation of postoperative complications in renal transplant recipients [1]. This radiotracer is extracted from the kidneys with high efficiency through secretion from proximal tubules $[2,3]$. The time-activity curves (TACs) of ${ }^{99 \mathrm{~m}} \mathrm{Tc}-\mathrm{MAG} 3$ renograms, which are produced by dynamic scan, enable the real-time and quantitative evaluation of multiple features of renal function, while the renography created by static scan provides spatial information about ${ }^{99 m}$ Tc-MAG3 kinetics and kidney morphology. The first phase of the TACs, which rises as ${ }^{99 m}$ Tc-MAG3 flows into renal tissue, can be used as an indicator of renal perfusion and tubular uptake, while the descending phase provides an assessment of tubular excretion [4,5] (Additional file 1). Thus, the measurement of intrarenal ${ }^{99}$ Tc-MAG3 kinetics provides a minimally invasive, simple, and quantitative test of renal function, which does not require blood or urine sampling. Given these features, ${ }^{99 \mathrm{~m}}$ Tc-MAG3 renography could serve as a useful tool for the repetitive and longitudinal assessment of renal function in pre-clinical animal models of renal disease. However, the application of this method to animal models of renal disease is currently limited, especially in rodents. In mice, only a few studies have employed it to assess ischemia and reperfusion renal injury [5,6]. Also, quantitative analysis and longitudinal investigation of ${ }^{99 m}$ Tc-MAG3 renograms have not been carried out in animals with unilateral ureteral obstruction (UUO).

Obstructive nephropathy is a major cause of renal impairment in infants and children [7]. Over the past few decades, the cellular and molecular events involved in obstructive renal injury have been elucidated by surgical ligation of a ureter (known as UUO) in postnatal and adult animals $[8,9]$. These include tubular and myofibroblast proliferation, interstitial inflammation, tubular atrophy/apoptosis, interstitial fibrosis, and reninangiotensin and TGF $\beta$ activation. Further, the UUO model recapitulates all of the key features of renal fibrosis, a hallmark of progressive renal disease; therefore, the model is widely used for the research of renal fibrogenic response $[9,10]$. However, disorders of renal function during the course of the UUO model are incompletely understood, as the measurements of renal function in a single kidney are methodologically limited. Thus, the purpose of the present study was to investigate the use of dynamic ${ }^{99 m}$ Tc-MAG3 imaging for the assessment of renal function in a mouse UUO model. Our data demonstrate that renal uptake of ${ }^{99 m}$ Tc-MAG3 is remarkably and rapidly reduced in UUO kidneys as early as day 1 post-surgery, while it is minimally changed in contralateral kidneys. Further, the parametric analysis of TACs suggested that renal perfusion as well as tubular uptake is reduced in UUO kidneys. This imaging technique should allow non-invasive assessments of UUO renal injury and enable a more rapid interrogation of novel therapeutic agents and protocols.

\section{Methods}

\section{Animals}

Male C57BL/6N mice, aged 6 weeks, were purchased from Harlan Laboratories (Indianapolis, IN). Animals were fed a standard diet and allowed free access to water. All animal procedures were conducted in accordance with institutional guidelines and approved by the Vanderbilt University Institution of Animal Care and Use Committee.

\section{Unilateral ureteral obstruction}

Mice were anesthetized with $2 \%$ isoflurane and the left kidney was exposed through the site of the left flank incision. The ureter was obstructed completely near the renal pelvis using a 4-0 silk tie suture at two points. Sham-operated mice underwent the same surgical procedure except for the ureter ligation. The mice were imaged at 0 (3 hours), 1, 3, and 6 days after surgery.

\section{${ }^{99 m}$ Tc-MAG3 Renal scintigraphy}

Mice were anesthetized with 1.5\%/98.5\% isoflurane/oxygen mixture and two mice at a time were placed directly on top of a parallel-hole collimator positioned over one of the four camera heads of a NanoSPECT/CT (Bioscan, Washington, DC). The NanoSPECT was set up in single-camera planar-dynamic mode. A 40 min dynamic acquisition was initiated simultaneously with retro-orbital injection of $\sim 37$ $\mathrm{MBq} / 0.2 \mathrm{~mL}$ of ${ }^{99 \mathrm{~m}} \mathrm{Tc}-\mathrm{MAG} 3$ (obtained from VUMC Radiopharmacy, Vanderbilt University). The $40 \mathrm{~min}$ dynamic planar acquisition was divided into 240 frames yielding a temporal resolution of $10 \mathrm{sec}$. Two-dimensional images were collected in a $256 \times 256$ matrix with a pixel size of $0.29 \mathrm{~mm} \times 0.29 \mathrm{~mm}$. For static imaging, three dimensional images were collected in a $176 \times 176 \times 112$ matrix with a pixel size of $0.2 \mathrm{~mm} \times 0.2 \mathrm{~mm} \times 0.2 \mathrm{~mm}$ through a 40-min scan (a temporal resolution of $40 \mathrm{~min}$ ), producing high resolution imaging data. In a comparison study (retro-orbital vs. intravenous injections), ${ }^{99} \mathrm{~m}$ TcMAG3 was injected into mice via jugular catheter. Jugular vein catheterization was carried out as described [11].

\section{Image analysis}

The planar images were imported into the medical image viewer software Amide [12]. Two-dimensional ellipsoidal shaped regions of interest (ROIs) were drawn 


\section{UUO Kidneys}

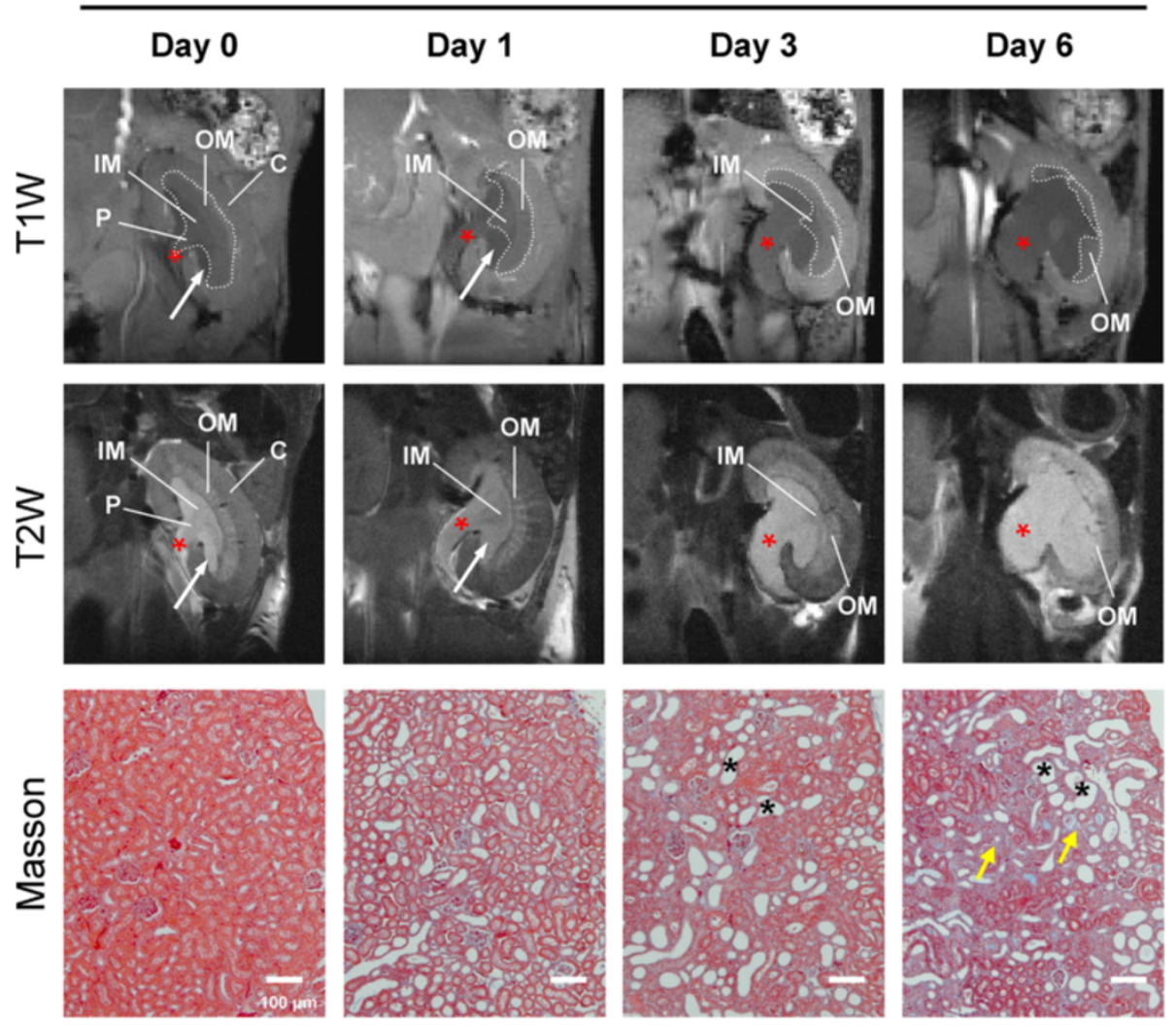

Figure 1 Renal structure and histopathology in UUO mice. (A) Representative T1- and T2-weighted coronal MRI images of UUO kidney at day 0, 1, 3 and 6 post obstruction. Renal pelvic space (asterisks) is progressively expanded in the obstructed kidney, and renal medulla (outlined with dotted lines), especially inner medulla and papilla, is remarkably decreased as the disease progresses. Arrows indicate urine. C, cortex; OM, outer medulla (inner stripe); IM, inner medulla; P, papilla. (B) Representative histological sections of mouse kidneys subjected to unilateral ureteral obstruction. Tubulointerstitial region increases markedly over 6 days in UUO kidneys, as indicated by destructive morphology of renal tubules (asterisks) and interstitial fibrosis (arrows). Bar=100 $\mu \mathrm{m}$. Masson trichrome stain.

around each kidney and a background region outside the mouse. The radioactivity within the ROIs was extracted for each frame and normalized to the injected dose for each animal, dividing image voxel values by the actual injected dose as measured in a CRC-15W dose calibrator (Capintec, Ramsey, NJ), to establish the TACs over the entire duration of the scan. The data were expressed as normalized image value (NIV). The slope of initial linear uptake (from 10s to 40s)(SIU) in units of $1 / \mathrm{s}$, peak activity in units of NIV, and the time to peak or plateau (TTP or TTPt) in units of seconds were computed from the renogram of each kidney $[5,13]$. [Note: Since the TACs of UUO kidneys plateau and do not peak, we measured the plateau activity and the time-toplateau for these kidneys.]

\section{Magnetic resonance imaging (MRI)}

Structural changes in UUO kidneys were assessed by MRI studies on a Varian $7 \mathrm{~T}$ horizontal bore imaging system (Lexington, MA). Anesthesia was induced and maintained

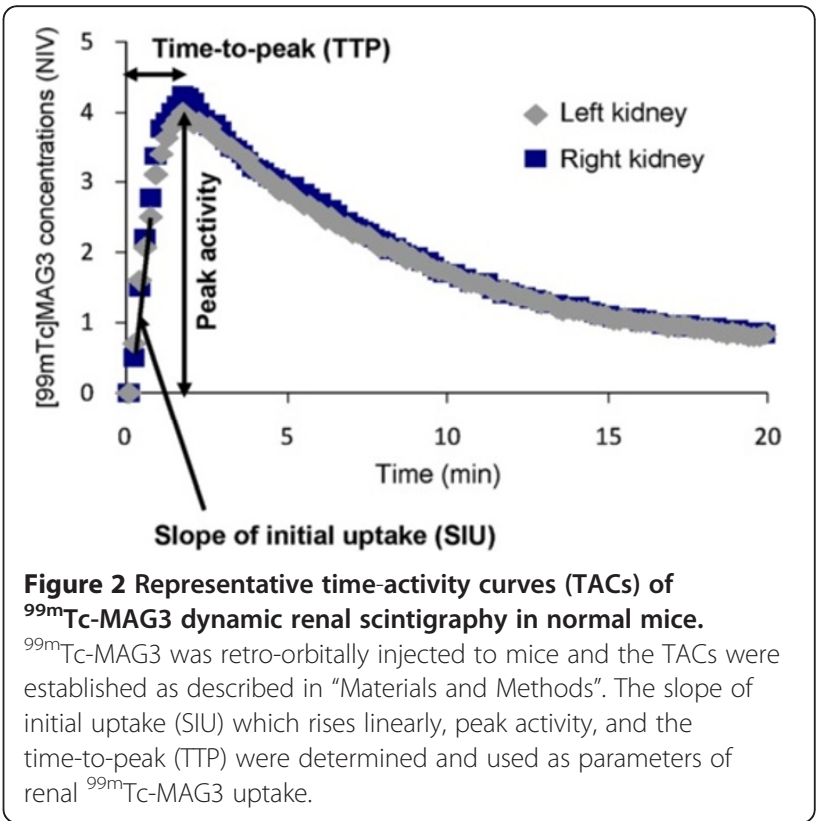



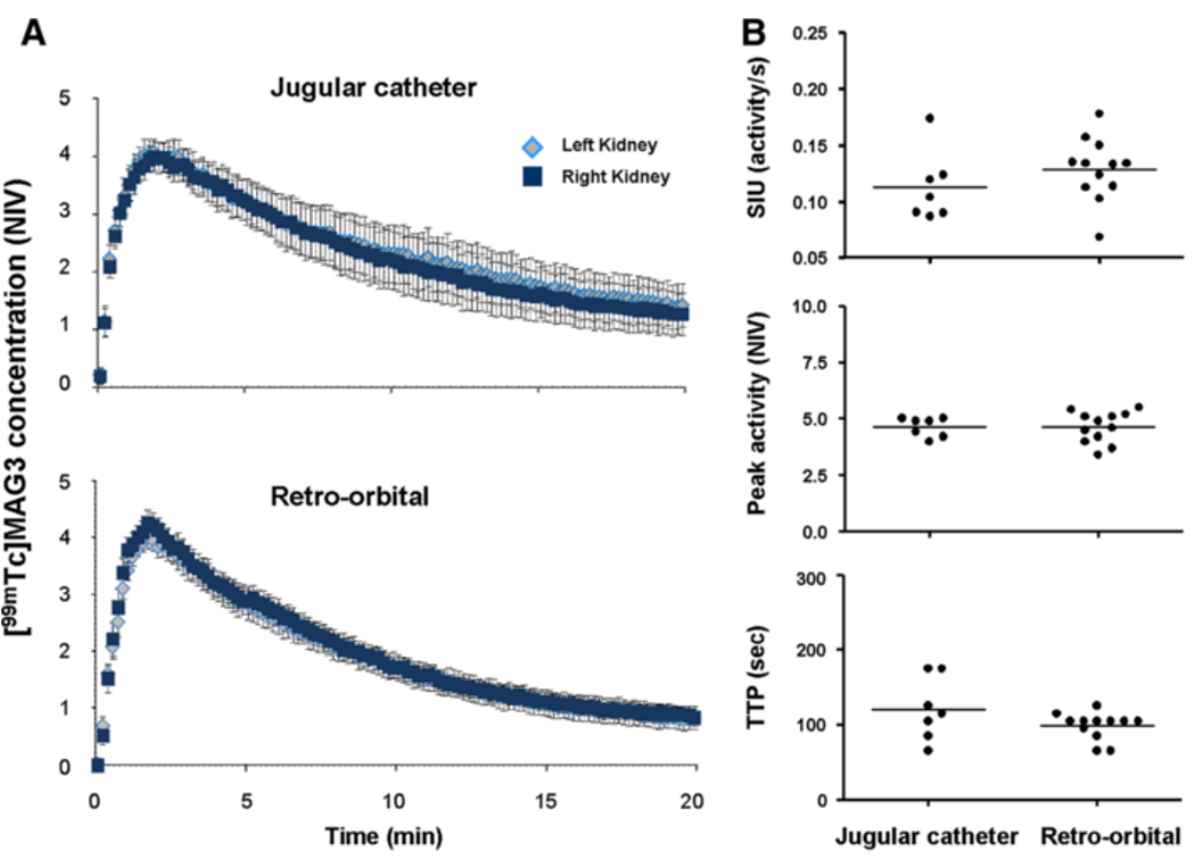

Figure 3 Comparison of TACs established by intravenous and retro-orbital ${ }^{99 \mathrm{~m}}$ Tc-MAG3 injections. (A) Mean TACs established by intravenous ( $n=7)$ and retro-orbital $(n=12){ }^{99 m}$ Tc-MAG3 injections. For intravenous injection, ${ }^{99 m}$ Tc-MAG3 was injected via jugular vein catheter. Values are means \pm SE. (B) Comparison of the parameters (SIU, peak activity, and TTP) extracted from the TACs generated by intravenous and retro-orbital ${ }^{99 m}$ TC-MAG3 injections. No significant difference was found between the two procedures.

with a $1.5 \% / 98.5 \%$ isoflurane/oxygen mixture. A constant body temperature of $37^{\circ} \mathrm{C}$ was maintained using heated air flow. Animals were placed in a prone position and imaged using a birdcage 25 -mm inner diameter radiofrequency (RF) coil. All imaging protocols were based on a field-of-view of $25.6 \times 25.6 \mathrm{~mm}^{2}$. A multi-slice gradient echo imaging sequence [repetition time $(\mathrm{TR})=25 \mathrm{~ms}$; echo time $(\mathrm{TE})=2.5 \mathrm{~ms} ; 128 \times 128$ matrix; 2 -mm slice thickness] was used to acquire 3-plane multi-slice images for proper positioning and slice selection. A coherent gradient echo sequence was used for T1-weighted imaging (TR = $45 \mathrm{~ms}$; $\mathrm{TE}=2.25 \mathrm{~ms}$; flip angle $=35^{\circ}$; number of excitations $(\mathrm{NEX})=81)$. T2 contrast was achieved with the use of a fast spin-echo (FSE) sequence (TR $=2000 \mathrm{~ms}$; effective $\mathrm{TE}=48 \mathrm{~ms}$; RARE-factor 8, $256 \times 256$ matrix; 0.5-mm slice thickness).

\section{Histological assessment}

Kidneys were removed from euthanized mice, fixed overnight in $10 \%$ buffered formalin, and paraffin tissue sections were stained with Masson trichrome using standard procedures.

\section{Statistical analysis}

One-way ANOVA followed by a Dunnett post test was carried out to compare the mean values in different days and groups. A two-tailed paired t-test was carried out for comparisons between obstructed and unobstructed (contralateral) kidneys. Data were expressed as means \pm SEM. The derived $p$-values were considered statistically significant when less than 0.05 .

\section{Results}

In the present study, we assessed renal function in UUO mice using ${ }^{99 \mathrm{~m}}$ Tc-MAG3 dynamic renal scintigraphy. Renal uptake of ${ }^{99 \mathrm{~m}}$ Tc-MAG3 was assessed by parametric analysis of TACs. Since ${ }^{99 \mathrm{~m}} \mathrm{Tc}-\mathrm{MAG} 3$ is not excreted from UUO kidneys, parametric analysis of ${ }^{99 \mathrm{~m}}$ Tc-MAG3 excretion was not performed in this study.

\section{Renal structure and histopathology in UUO mice}

First, we confirmed generation and severity of UUO model by MRI and histological investigation. Figure 1 shows representative T1- and T2-weighted MR images of a UUO mouse at $0,1,3$ and 6 days post-surgery. The renal pelvic space was gradually expanded in obstructed kidneys as early as 1 day post ureteral ligation and the components of renal medulla, especially inner medulla and papilla, were remarkably decreased as the disease progressed. Reduction of renal cortex was also observed at day 3 and day 6 post obstruction. Histological investigation displayed typical patterns of UUO renal disease, including tubular dilatation, flattening and atrophy, and expansion of interstitial area with extracellular matrix accumulation and infiltration of inflammatory cells (Figure 1, bottom panels). Tubulointerstitial region progressed markedly over 6 days 
after ureteral obstruction. Obvious structural changes were not observed in contralateral kidneys and shamoperated mouse kidneys (data not shown).

\section{Parametric analysis of TACs and comparison of intravenous and retro-orbital ${ }^{99 \mathrm{~m}}$ Tc-MAG3 injections}

Renal function was assessed in UUO kidneys based on the TACs and their parametric analysis. Three parameters were measured for individual kidneys: 1) the slope of initial linear uptake (SIU, 1/s), which is related to renal perfusion; 2) the peak activity (NIV); and 3) the time-to-peak or plateau (TTP or TTPt, sec) (Figure 2). Radiotracers are usually administrated via the tail vein in rodents. However, repeated injections are difficult as the procedure frequently causes soft tissue extravasations and tail scars, preventing subsequent injections. Repeatable and proper intravenous injections are fundamental for the longitudinal and quantitative analysis of ${ }^{99} \mathrm{~m}$ TcMAG3 renal scintigraphy. Therefore, we first compared retro-orbital and intravenous injections, given the facts that the former is much easier to accomplish at multiple time points and that retro-orbital injection was recently shown to be an effective route of radiotracer administration during PET studies in mice [14]. As shown in Figure $3 \mathrm{~A}$, the TACs of ${ }^{99 \mathrm{~m}}$ Tc-MAG3 renal scintigraphy obtained from retro-orbital injections were comparable to those from intravenous injections via jugular vein catheter. There was no significant difference in the SIU, peak activity, and TTP parameters between these two procedures (Figure 3B). In addition, a comparative study using UUO day 1 mice also showed no difference between these two administration routes (data not shown). These results demonstrate that retro-orbital injection can be used for ${ }^{99 \mathrm{~m}} \mathrm{Tc}-\mathrm{MAG} 3$ renal scintigraphy in mice. Therefore, in this study we assessed renal function in UUO mice using retro-orbital injections of ${ }^{99 \mathrm{~m}}$ Tc-MAG3.

\section{Assessment of renal function in UUO mice using ${ }^{99 m}$ Tc-MAG3 dynamic scintigraphy}

Renal scintigraphy was carried out in UUO and shamoperated mice at 0 ( 3 hours), 1, 3, and 6 days after surgery. Non-surgery mice were also imaged as a control. Figure 4 A-D shows TACs of UUO kidneys at each time point. As shown in Figure $4 \mathrm{~B}$, renal uptake of ${ }^{99 \mathrm{~m}} \mathrm{Tc}$ MAG3 was remarkably reduced in UUO kidneys as early

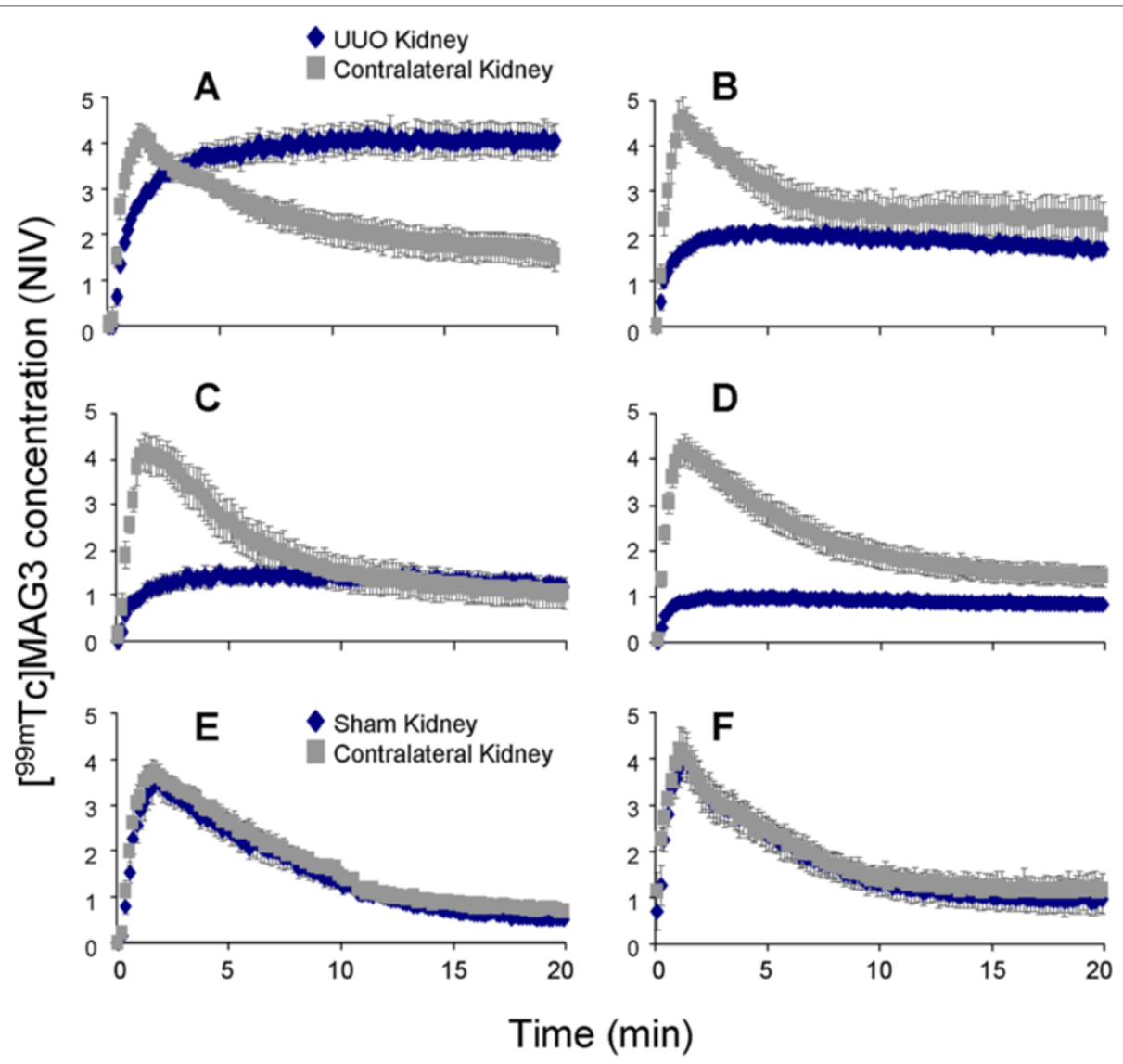

Figure 4 TACs of ${ }^{99 m}$ Tc-MAG3 dynamic renal scintigraphy in UUO mice. (A) UUO day 0, (B) UUO day 1, (C) UUO day 3, (D) UUO day 6, (E) Sham operation day 0, (F) Sham operation day 6. Values are means \pm SE. The numbers of mice are described in Table 1. 
as day 1 post obstruction, as indicated by low total activity throughout the TACs, and it was further decreased as the disease progressed (Figure 4C and 4D). The TACs in UUO kidneys plateaued over time, indicating that ${ }^{99 m}$ Tc-MAG3 is not excreted in these kidneys. Profound reduction of ${ }^{99 \mathrm{~m}}$ Tc-MAG3 uptake was also demonstrated in UUO kidney by the time interval images of renal scintigraphy (Figure 5). Further, static renal scintigraphy demonstrated that excretion of ${ }^{99 m}$ Tc-MAG3 to renal pelvis is highly limited in UUO kidneys, even at day 0 (3 hrs) post surgery (Figure 6); the finding indicates that TACs of UUO kidneys mostly demonstrate the deposition of ${ }^{99 m}$ Tc-MAG3 in renal parenchyma. The contralateral kidneys in UUO mice (Figure 4 A-D) and kidneys in sham-operated mice (Figure 4E and 4F) showed similar TACs. Table 1 and Figure 7 show the parametric analysis of ${ }^{99 m}$ Tc-MAG3 renal scintigraphy based on TACs obtained from UUO and sham operation mice at day $0,1,3$, and 6 post surgery. The results of statistical analysis are shown in Table 2 . As compared to sham-operated kidneys, SIU was remarkably (> 60\%) decreased in UUO kidneys as early as day 1 post obstruction, while contralateral kidneys showed SIU values comparable to those in sham-operated kidneys (Figure 7A). It is noteworthy that the slope of the ascending phase (50s to peak, denoted Phase 2), which follows the initial linear uptake of ${ }^{99 \mathrm{~m}}$ Tc-MAG3 (10s to 40s, denoted Phase 1), was progressively increased in contralateral kidneys, though it was not statistically significant against day 0 or sham operation kidneys (Figure 8). The plateau activity of TACs was also remarkably ( 65\%) reduced in UUO kidneys as early as day 1 post obstruction and it declined further as the disease progressed, while the peak activity in contralateral kidneys was comparable to those in sham-operated kidneys during the study period (Figure 7B). Further, the time-to-plateau (TTPt) in UUO kidneys largely exceeded the time-to-peak (TTP) values in sham-operated or contralateral kidneys (Figure 7C), suggesting that ${ }^{99 \mathrm{~m}}$ Tc-MAG3 is slowly up-taken in UUO kidneys. The TTP was unaltered in contralateral kidneys as compared with sham operation kidneys, yet contralateral kidneys at UUO day 6 showed significantly shorter TTP than at UUO day 0,1 , and 3 (Figure $7 \mathrm{C}$, Table 2). There was no significant difference in the peak:10min ratio, an indicator of tubular excretion rate $[5,6]$, between contralateral kidneys and sham-operated kidneys, though contralateral kidneys showed lower peak:10min ratios at day 0 and day 1 , perhaps due to intravascular fluid shifts during surgical procedure (data not shown). No difference was observed in SIU, peak activity, TTP, and peak:10min
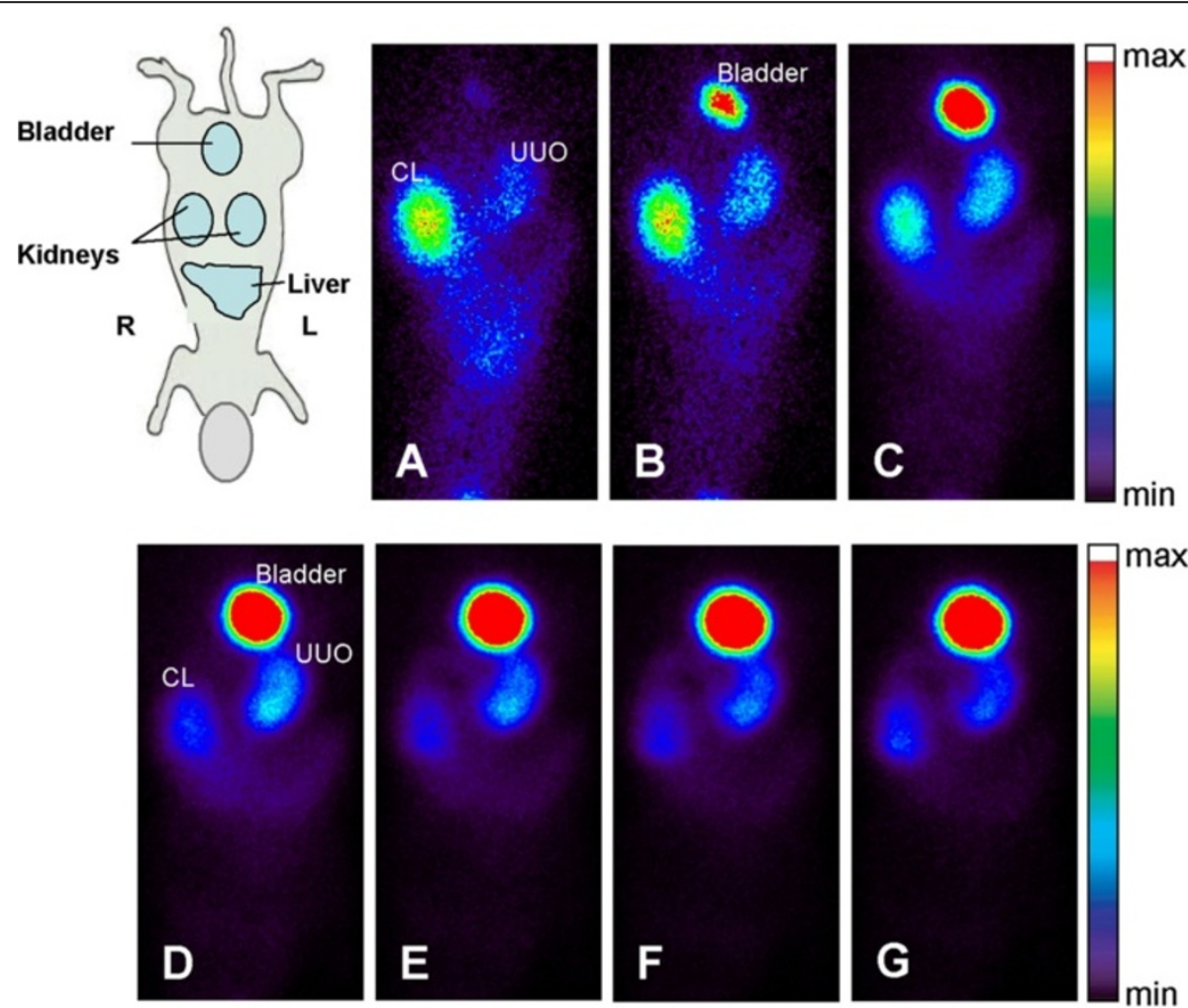

Figure 5 Representative time-interval images of ${ }^{99 m}$ Tc-MAG3 dynamic renal scintigraphy in UUO mice at 6 days post obstruction. Uptake of the tracer is remarkably reduced in UUO kidney. A) 0-1 min, B) 1-2 min, C) 2-6 min, D) 6-15 min, E) $15-25 \mathrm{~min}$, F) 25-35 min, and G) 35-40 min. R, right; L, left; CL, contralateral kidney. 

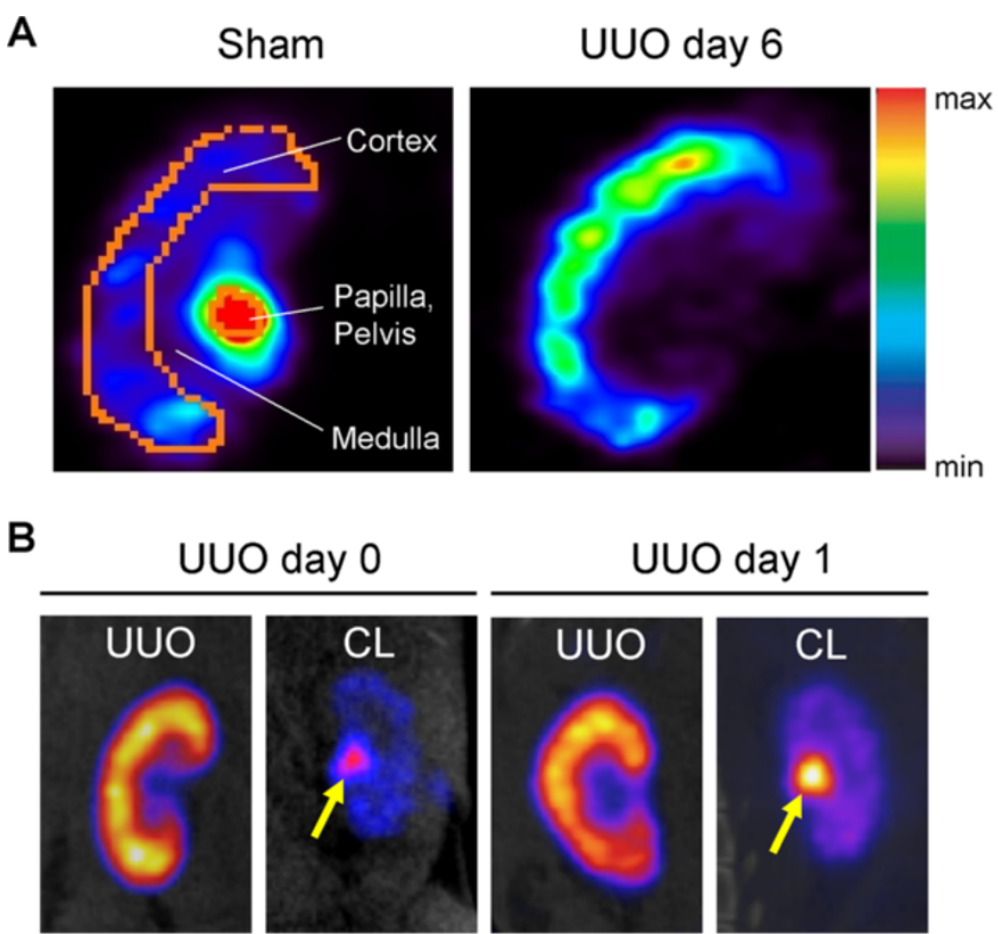

Figure 6 Static ${ }^{99 m}$ Tc-MAG3 imaging of UUO mice kidneys. A) Static SPECT images (a 40-min acquisition) were obtained from UUO day 6 and sham-operated mice kidneys after retro-orbital injection of ${ }^{99 \mathrm{~m}} \mathrm{Tc}-\mathrm{MAG}$. The excretion of ${ }^{99 \mathrm{~m}} \mathrm{Tc}$-MAG3 to renal pelvis is not observed in UUO day 6 kidney, while ${ }^{99 \mathrm{~m}} \mathrm{Tc}$-MAG3 gets into renal pelvis in sham-operated kidney during a 40 min scan. B) Similar findings were observed in UUO day 0 and day 1 kidneys, while ${ }^{99 \mathrm{~m} T C-M A G 3}$ was excreted to renal pelvis (arrows) in contralateral kidneys (CL). Representative images are shown.

ratio between sham-operated and non-surgery mice kidneys and between sham-operated and contralateral kidneys in sham mice.

\section{Discussion}

In the present study, we have applied ${ }^{99 \mathrm{~m}}$ Tc-MAG3 renal scintigraphy to a mouse model of obstructive nephropathy and non-invasively evaluated renal function over 6 days post obstruction. Our results demonstrate that renal uptake of ${ }^{99 \mathrm{~m}}$ Tc-MAG3 is rapidly reduced by ureteral ligation, while it is minimally changed in the contralateral kidney. This work provides the first non-invasive evaluation of longitudinal changes in renal function in UUO animals. Further, our data demonstrate that retro-orbital

Table 1 Parameters extracted from the TACs of ${ }^{99 m}$ Tc-MAG3 dynamic renal scintigraphy

\begin{tabular}{lcccc}
\hline Kidney & $\mathbf{n}$ & Peak or plateau* activity (NIV) & SIU (activity/s) & TTP, TTPt* (s) \\
\hline Normal (non-surgery) & 12 & $4.63 \pm 0.20$ & $0.129 \pm 0.008$ & $98 \pm 5$ \\
UUO day 0 & 6 & $4.48 \pm 0.19$ & $0.090 \pm 0.010$ & $437 \pm 35$ \\
UUO day 1 & 5 & $1.98 \pm 0.20$ & $0.051 \pm 0.014$ & $213 \pm 38$ \\
UUO day 3 & 6 & $1.45 \pm 0.10$ & $0.055 \pm 0.011$ & $258 \pm 46$ \\
UUO day 6 & 8 & $1.06 \pm 0.09$ & $0.041 \pm 0.008$ & $138 \pm 33$ \\
Contralateral day 0 & 6 & $4.22 \pm 0.26$ & $0.139 \pm 0.015$ & $93 \pm 5$ \\
Contralateral day 1 & 5 & $4.56 \pm 0.36$ & $0.122 \pm 0.012$ & $91 \pm 9$ \\
Contralateral day 3 & 6 & $4.43 \pm 0.35$ & $0.135 \pm 0.018$ & $95 \pm 14$ \\
Contralateral day 6 & 8 & $4.43 \pm 0.28$ & $0.136 \pm 0.014$ & $83 \pm 5$ \\
Sham day 0 & 7 & $4.19 \pm 0.33$ & $0.131 \pm 0.032$ & $101 \pm 10$ \\
Sham day 1 & 8 & $4.95 \pm 0.40$ & $0.158 \pm 0.023$ & $108 \pm 18$ \\
Sham day 3 & 5 & $4.16 \pm 0.18$ & $0.125 \pm 0.009$ & $87 \pm 17$ \\
Sham day 6 & 4 & $4.35 \pm 0.49$ & $0.149 \pm 0.027$ & $85 \pm 7$ \\
\hline Vals ar & & &
\end{tabular}

Values are means \pm SE. * Plateau activity and the time-to-plateau (TTPt) were measured in UUO kidneys. 


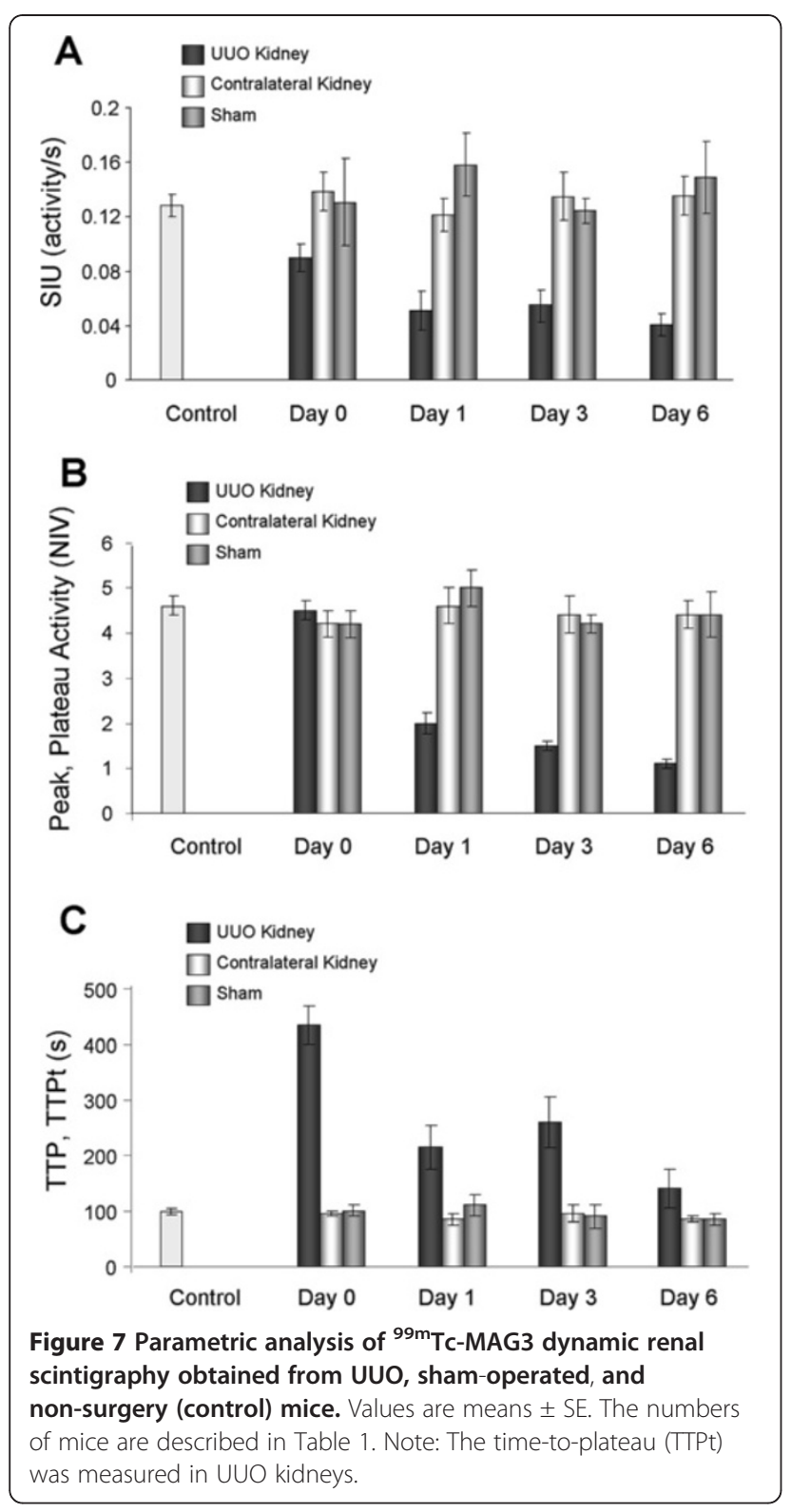

Table 2 Summary of statistical tests between obstructed, contralateral, and sham-operated kidneys on all days post UUO/sham surgery

\begin{tabular}{|c|c|c|c|}
\hline \multirow[t]{2}{*}{ Test } & \multicolumn{3}{|c|}{$P<0.05$} \\
\hline & Peak activity & SIU & TTP \\
\hline UUO vs. Sham & days $1,3,6$ & days $1,3,6$ & $N / A^{*}$ \\
\hline Contralateral vs. Sham & none & none & none \\
\hline UUO vs. Contralateral & days $1,3,6$ & days $0,1,3,6$ & $N / A^{*}$ \\
\hline UUO vs. UUO & day 0 vs. days $1,3 \& 6$; day 1 vs. day 6 & day 0 vs. day 6 & day 0 vs. days $1,3 \& 6$ \\
\hline Contralateral vs. Contralateral & none & none & day 6 vs. days $0,1, \& 3$ \\
\hline
\end{tabular}

* As TACs of UUO kidney did not peak, statistical test was not applied to this section. injection can be used for ${ }^{99 \mathrm{~m}}$ Tc-MAG3 renal scintigraphy in mice. kidney. Several studies have demonstrated acute reduction in renal blood flow (RBF) in UUO animals. Vaughan et al. measured RBF in a dog UUO model using a flow probe placed in renal artery and showed that RBF is transiently increased in UUO kidney at $0-1.5$ hrs post obstruction, followed by a progressive decline $[15,16]$. In their study, RBF was decreased by 50\% in UUO kidney within $18 \mathrm{hrs}$ post obstruction, while changes in RBF were not observed in contralateral kidney. Similar results have been demonstrated by other studies measuring renal arterial blood flow in rat or dog UUO models [17-19]. Thus, the changes of SIU are consistent with these previous results of RBF measurements on renal artery in experimental UUO kidneys, suggesting that the decreases in SIU may largely result from reduced renal perfusion. It is of note that the plateau activity is also substantially reduced in UUO mouse kidney at day 1 post obstruction, whereas a reduction of vessel density is not observed at this time point; the finding suggests that the capacity of ${ }^{99 \mathrm{~m}}$ Tc-MAG3 uptake is also reduced in UUO day 1 kidney, perhaps due to tubular cell injury. This may also be the reason why the time-to-plateau is shortened in UUO kidney at day 1 post obstruction. Thus, our results suggest that reduced renal perfusion and tubular injury causes remarkable reduction of ${ }^{99 \mathrm{~m}}$ Tc-MAG3 uptake in UUO kidney and that these parameters may be used for assessing the severity of obstructive renal disease.

In contrast to the obstructed kidney, the changes in SIU, peak activity, and TTP were not observed in contralateral kidney, even though the obstructed kidney was functionally dead. Given the fact that unilateral nephrectomy increases renal arterial blood flow by $~ 50 \%$ in two days $[20,21]$, it seems that the systemic factors produced by the UUO kidney and/or the presence of renal blood flow in the obstructed kidney attenuate adaptive responses in the contralateral kidney. It is of note that the slope of the ascending phase after linear uptake of ${ }^{99 \mathrm{~m}}$ Tc-MAG3 was increased in contralateral kidneys. Although the mechanism of this is currently unknown, the finding suggests that
A substantial reduction of SIU was observed in UUO 

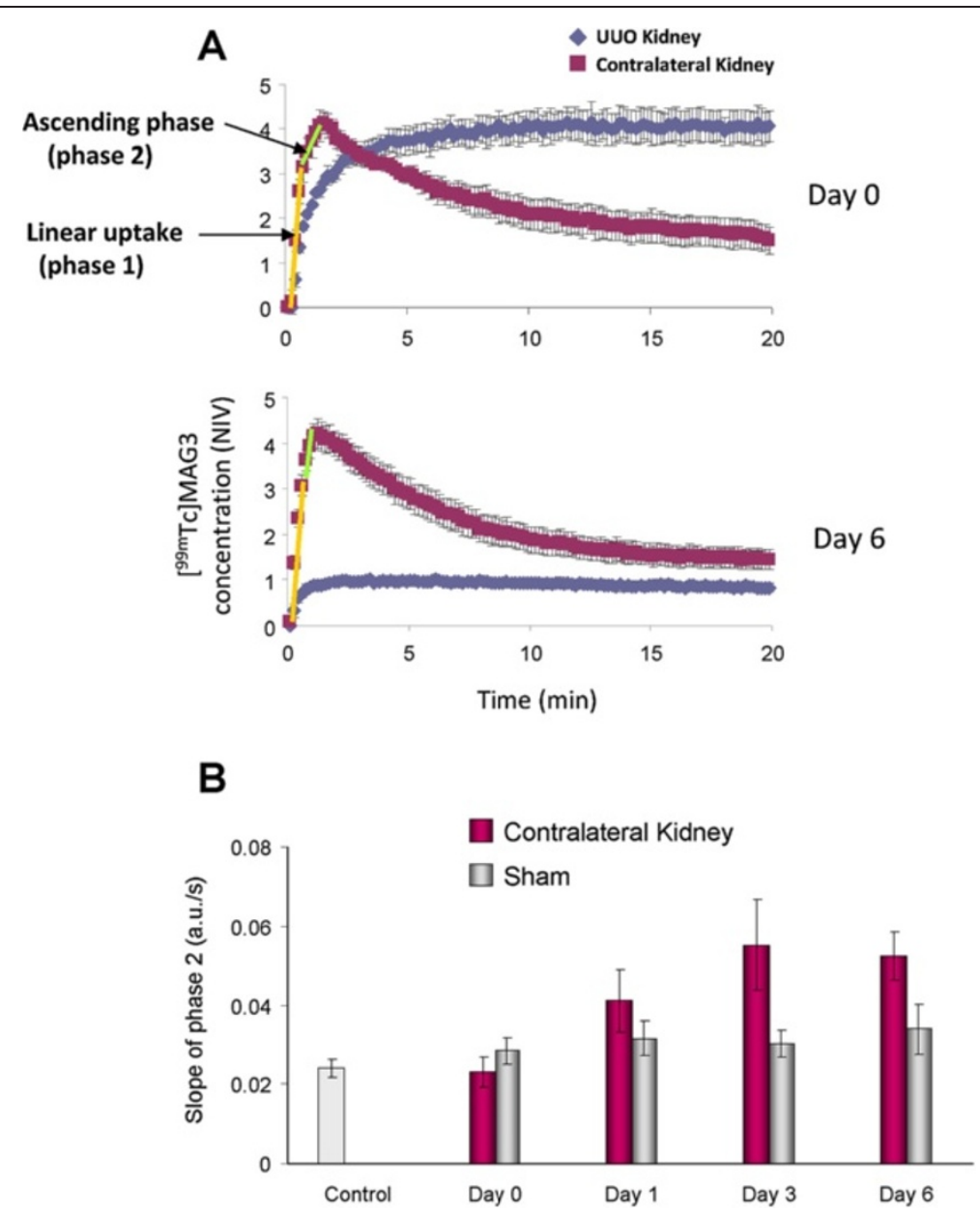

Figure 8 Slope of ascending phase is increased in contralateral kidneys after UUO surgery. A) Graphical depiction of ascending phase window selection in contralateral kidneys at 0 and 6 days post ureteral obstruction. B) The slope of ascending phase (from 50s to peak) was measured for the contralateral kidneys in UUO and sham-operated mice. Values are means \pm SE. The numbers of mice are described in Table 1.

early tubular excretion of ${ }^{99 \mathrm{~m}} \mathrm{Tc}-\mathrm{MAG} 3$ may be decreased in contralateral kidneys.

In dog and rat UUO models, it has been suggested that increased renal endothelin-1 production [18], enhanced renin-angiotensin system (RAS) [22], and insufficient nitric oxide synthesis $[15,16,23]$ cause vasoconstriction in obstructed kidney, and these alterations can increase renal vascular resistance, especially pre-glomerular resistance, resulting in reduction of renal arterial blood flow. Pharmacological agents to antagonize endothelin or RAS system, $\mathrm{L}$-arginine to promote nitric oxide production, and a calcium channel blocker (verapamil) which suppresses vasoconstriction were shown to be effective in increasing renal arterial blood flow in UUO kidney [16,18,19,22-24]. It would be of interest to determine the roles of these pathways in reduction of ${ }^{99 \mathrm{~m}} \mathrm{Tc}-\mathrm{MAG} 3$ uptake in a mouse UUO model using this imaging technique and genetically manipulated mice or pharmacological treatments. Also, further investigation would be required to determine the changes of renal perfusion in a mouse UUO model using a nuclear or MR imaging technique (e.g. dynamic susceptibility contrast MRI) and evaluate the relation between renal perfusion and the uptake of ${ }^{99 \mathrm{~m}} \mathrm{Tc}-\mathrm{MAG} 3$. Further, it is of note that tracer deposition within renal pelvis is highly limited in UUO mouse kidney, even at day 0 ( $3 \mathrm{hrs)}$ post surgery. Similar findings were also reported in pig UUO kidney [25], These findings suggest that ureteral obstruction immediately reduces tubular function and the radiotracer does not get into the renal pelvis. Further investigation would be required to determine the mechanism of this. Last, compared to the obstructed kidney, much less is known about the adaptive responses and mechanisms in contralateral kidney. This issue should also be addressed in future study.

\section{Conclusions}

This work assessed renal function in UUO mice by dynamic SPECT imaging with retro-orbital injection of 
${ }^{99 \mathrm{~m}}$ Tc-MAG3. Our data demonstrate that renal uptake of ${ }^{99 m}$ Tc-MAG3 is remarkably and rapidly reduced in UUO kidneys, while the changes are minimal in contralateral kidneys. The parametric analysis of TACs suggested that renal perfusion as well as tubular uptake is reduced in UUO kidneys. Given the fact that similar changes are observed in ${ }^{99 m}$ Tc-MAG3 renogram in human patients with renal obstruction [26,27], combination of this imaging technique with mouse UUO models should provide an effective assay for evaluating treatment protocols of this disease or screening new pharmacological agents as well as for elucidating pathophysiological mechanisms of obstructive kidney disease.

\section{Additional file}

Additional file 1: Tantawy MN et al Supplemental Figure S1: A supplemental figure demonstrating the phases of ${ }^{99 \mathrm{~m}} \mathrm{Tc}-\mathrm{MAG} 3$ TACs and their determinants.

\section{Abbreviations}

MAG3: Mercaptoacetyltriglycine; UUO: Unilateral ureteral obstruction; TACs: Time-activity curves; RBF: Renal blood flow; TTP: The time-to-peak; TTPt: The time-to-plateau; SPECT: Single photon emission-computed tomography; MRI: Magnetic resonance imaging.

\section{Competing interests}

The authors have no competing interests to declare.

\section{Authors' contributions}

M.N.T., C.C.Q., and T.T. provided conception and design of research; M.N.T. and F.W. performed SPECT and MR imaging; R.J., K.T., and C.M.H. performed UUO and sham surgery; R.J., K.T., and H.F. performed histological analysis; M. N.T., D.Z., and T.E.P. analyzed data; M.N.T., R.C.H., C.C.Q., and T.T. interpreted results of experiments; M.N.T. and T.T. prepared figures and drafted the manuscript; T.E.P., R.C.H., C.C.Q., and T.T. edited and finalized the manuscript All authors read and approved the final manuscript.

\section{Acknowledgments}

The authors thank Clare Osborne and Masayo Hino for technical assistance. This study was facilitated by the Vanderbilt Mouse Metabolic Phenotyping Center, O'Brien Mouse Kidney Physiology and Disease Center, and Pathology Core. This work was supported by National Institute of Diabetes and Digestive and Kidney Disease Grant P30 DK79341 (to R.C.H., T.T., and C.C.Q.)

\section{Author details}

${ }^{1}$ Radiology and Vanderbilt University Institute of Imaging Science, Nashville, TN, USA. ${ }^{2}$ Vanderbilt O'Brien Mouse Kidney Physiology and Disease Center, Vanderbilt University Medical Center, Nashville, TN, USA. ${ }^{3}$ Division of Endocrinology, Metabolism and Geriatric Medicine, Akita University Graduate School of Medicine, Akita, Japan. ${ }^{4}$ Division of Nephrology and Hypertension, Vanderbilt University School of Medicine, S-3223, MCN, Nashville, TN 37232 USA.

Received: 14 June 2012 Accepted: 27 November 2012

Published: 10 December 2012

\section{References}

1. Itoh K: $99 m$ mc-MAG3: review of pharmacokinetics, clinical application to renal diseases and quantification of renal function. Ann Nucl Med 2001, 15(3):179-190

2. Fritzberg AR, Kasina S, Eshima D, Johnson DL: Synthesis and biological evaluation of technetium-99m MAG3 as a hippuran replacement. J NuCl Med 1986, 27(1):111-116.
3. Gates GF: Filtration fraction and its implications for radionuclide renography using diethylenetriaminepentaacetic acid and mercaptoacetyltriglycine. Clin Nucl Med 2004, 29(4):231-237.

4. Esteves FP, Taylor A, Manatunga A, Folks RD, Krishnan M, Garcia EV: 99mTc-MAG3 renography: normal values for MAG3 clearance and curve parameters, excretory parameters, and residual urine volume. AJR Am J Roentgenol 2006, 187(6):W610-W617.

5. Roberts J, Chen B, Curtis LM, Agarwal A, Sanders PW, Zinn KR: Detection of early changes in renal function using $99 m$ mc-MAG3 imaging in a murine model of ischemia-reperfusion injury. Am J of Physiol Renal Physiol 2007, 293(4):F1408-F1412

6. Herrler T, Tischer A, Meyer A, Feiler S, Guba M, Nowak S, Rentsch M, Bartenstein $\mathrm{P}$, Hacker $\mathrm{M}$, Jauch KW: The intrinsic renal compartment syndrome: new perspectives in kidney transplantation. Transplantation 2010, 89(1):40-46.

7. Collins AJ, Foley R, Herzog C, Chavers B, Gilbertson D, Ishani A, Kasiske B, Liu J, Mau LW, McBean M, et al: Excerpts from the United States Renal Data System 2007 annual data report. Am J Kidney Dis 2008, 51(Suppl 1):A6-A7.

8. Chevalier RL, Thornhill BA, Forbes MS, Kiley SC: Mechanisms of renal injury and progression of renal disease in congenital obstructive nephropathy. Pediatr Nephrol 2010, 25(4):687-697.

9. Eddy AA, Lopez-Guisa JM, Okamura DM, Yamaguchi I: Investigating mechanisms of chronic kidney disease in mouse models. Pediatr Nephrol 2012, 27(8):1233-1247.

10. Chevalier RL, Forbes MS, Thornhill BA: Ureteral obstruction as a model of renal interstitial fibrosis and obstructive nephropathy. Kidney Int 2009, 75(11):1145-1152

11. Ayala JE, Bracy DP, Malabanan C, James FD, Ansari T, Fueger PT, McGuinness $\mathrm{OP}$, Wasserman DH: Hyperinsulinemic-euglycemic clamps in conscious, unrestrained mice. J Vis Exp 2011, 57:e3188.

12. Loening AM, Gambhir SS: AMIDE: a free software tool for multimodality medical image analysis. Mol Imaging 2003, 2(3):131-137.

13. Mustafa S, Elgazzar AH, Essam H, Gopinath S, Mathew M: Hyperthermia alters kidney function and renal scintigraphy. Am J Nephrol 2007, 27(3):315-321

14. Nanni C, Pettinato C, Ambrosini V, Spinelli A, Trespidi S, Rubello D, Al-Nahhas A, Franchi R, Alavi A, Fanti S: Retro-orbital injection is an effective route for radiopharmaceutical administration in mice during small-animal PET studies. Nucl Med Commun 2007, 28(7):547-553.

15. Moody TE, Vaughn ED Jr, Gillenwater JY: Relationship between renal blood flow and ureteral pressure during 18 hours of total unilateral uretheral occlusion. Implications for changing sites of increased renal resistance. Invest Urol 1975, 13(3):246-251.

16. Vaughan ED Jr, Marion D, Poppas DP, Felsen D: Pathophysiology of unilateral ureteral obstruction: studies from Charlottesville to New York. J Urol 2004, 172:2563-2569.

17. Yeh $\mathrm{CH}$, Chiang HS, Lai TY, Chien CT: Unilateral ureteral obstruction evokes renal tubular apoptosis via the enhanced oxidative stress and endoplasmic reticulum stress in the rat. Neurourol Urodyn 2011 30(3):472-479.

18. Kahn SA, Gulmi FA, Chou SY, Mooppan UM, Kim H: Contribution of endothelin-1 to renal vasoconstriction in unilateral ureteral obstruction: reversal by verapamil. J Urol 1997, 157(5):1957-1962.

19. Bhangdia DK, Gulmi FA, Chou SY, Mooppan UM, Kim H: Alterations of renal hemodynamics in unilateral ureteral obstruction mediated by activation of endothelin receptor subtypes. J Urol 2003, 170(5):2057-2062.

20. Sigmon DH, Gonzalez-Feldman E, Cavasin MA, Potter DL, Beierwaltes WH: Role of nitric oxide in the renal hemodynamic response to unilateral nephrectomy. J Am Soc Nephrol 2004, 15(6):1413-1420.

21. Valdivielso JM, Perez-Barriocanal F, Garcia-Estan J, Lopez-Novoa JM: Role of nitric oxide in the early renal hemodynamic response after unilateral nephrectomy. Am J Physiol 1999, 276:R1718-R1723.

22. Klahr S, Morrissey J: Obstructive nephropathy and renal fibrosis. Am J Physiol Renal Physiol 2002, 283(5):E861-F875.

23. Hegarty NJ, Young LS, Kirwan CN, O'Neill AJ, Bouchier-Hayes DM, Sweeney $P$. Watson RW, Fitzpatrick JM: Nitric oxide in unilateral ureteral obstruction: effect on regional renal blood flow. Kidney Int 2001 59(3):1059-1065.

24. Topcu SO, Celik S, Erturhan S, Erbagci A, Yagci F, Ucak R: Verapamil prevents the apoptotic and hemodynamic changes in response to unilateral ureteral obstruction. Int J Urol 2008, 15(4):350-355. 
25. Schlotmann A, Clorius JH, Rohrschneider WK, Clorius SN, Amelung F, Becker $K$ : Diuretic renography in hydronephrosis: delayed tissue tracer transit accompanies both functional decline and tissue reorganization. J Nucl Med 2008, 49(7):1196-1203.

26. Ozker K, Onsel C, Kabasakal L, Sayman HB, Uslu I, Bozluolcay S, Cansiz T, Kapicioglu T, Urgancioglu I: Technetium-99m-N, N-ethylenedicysteine-a comparative study of renal scintigraphy with technetium-99m-MAG3 and iodine-131-OIH in patients with obstructive renal disease. J Nucl Med 1994, 35(5):840-845.

27. Coffey J: Flow rate models in renal obstruction. World J Urol 2010, 29(1):109-114

doi:10.1186/1471-2369-13-168

Cite this article as: Tantawy et al: Assessment of renal function in mice with unilateral ureteral obstruction using ${ }^{99 \mathrm{~m}} \mathrm{Tc}-\mathrm{MAG} 3$ dynamic scintigraphy. BMC Nephrology 2012 13:168.

\section{Submit your next manuscript to BioMed Central and take full advantage of:}

- Convenient online submission

- Thorough peer review

- No space constraints or color figure charges

- Immediate publication on acceptance

- Inclusion in PubMed, CAS, Scopus and Google Scholar

- Research which is freely available for redistribution 